\title{
Larvicultura de Trairão (Hoplias lacerdae) em Água Doce e Água Salinizada
}

\author{
Ronald Kennedy Luz ${ }^{1}$, Maria Célia Portella ${ }^{2}$
}

\begin{abstract}
RESUMO - O experimento foi conduzido com o objetivo de desenvolver técnicas de manejo durante a larvicultura de trairão, realizando cultivos em água doce ou ligeiramente salinizada, utilizando náuplios de Artemia como alimento vivo. No início da alimentação exógena, oito dias após a eclosão, as larvas foram contadas e estocadas em 12 recipientes com volume útil de 1,5 L cada, dotados de sistema de aeração, numa densidade de 10 larvas/L, distribuídos dentro de três tanques de $130 \mathrm{~L}$, contendo água com temperatura controlada $\left(29,5^{\circ} \mathrm{C}\right)$. Os tanques foram totalmente cobertos com lona plástica preta, mantendo o ambiente interno escuro, e descobertos somente para os manejos diários. As larvas foram submetidas a três tratamentos: cultivo em água doce; em água a 2\%o de salinidade; e em água a 4\%o de salinidade. Cada tratamento teve quatro repetições. A alimentação foi fornecida nas proporções diárias de 300 náuplios de Artemial larva, do primeiro ao quinto dia, de 600 náuplios de Artemia/larva, do sexto ao décimo dia e de 900 náuplios de Artemia/larva, do décimo primeiro ao décimo quinto dia, divididos em três refeições. Ao final do experimento, foram avaliadas a sobrevivência, o crescimento (comprimento e peso), a taxa de crescimento específico e a taxa de resistência ao estresse. Após 15 dias de tratamento, não foram verificadas diferenças significativas entre os resultados das variáveis analisadas. As condições de cultivo mostraram-se eficientes, possibilitando altas taxas de sobrevivência (valores médios superiores a 91,6\%), sendo a Artemia um alimento atrativo. Os níveis de salinidade utilizados não afetaram o desenvolvimento das larvas e alevinos.
\end{abstract}

Palavras-chave: trairão, Hoplias lacerdae, larvicultura, salinidade, canibalismo, Artemia

\section{Trairao (Hoplias lacerdae) Larviculture in Slightly Saline Freshwater}

\begin{abstract}
The objective of this work was the development of larviculture handling technics to rear trairao in slightly saline freshwater, with Artemia nauplii as live food. At the beginning of exogenous feeding, eight days after hatching, trairao larvae were counted and stocked in twelve 1.5-L vessels, equipped with aeration, at 10 larvae/L density. The vessels were distributed in three 130-L tanks filled with controlled temperature water $\left(29.5^{\circ} \mathrm{C}\right)$. The tanks were covered with black plastic to keep the environment dark, and uncovered only for daily management. The larvae were submitted to three treatments with four replicates, as follows: freshwater culture; water with $2 \%$ salinity; and water with $4 \%$ salinity. For the first five days, larvae were fed 300 Artemia nauplii/larvae. In the subsequent five days, larvae were fed 600 Artemia nauplii/larvae, and from the $11^{\text {th }}$ to the $15^{\text {th }}$ day, 900 Artemia nauplii/larvae, divided in three portions. At the end of the experiment, survival, growth (length and weight), specific growth rates and stress resistence rate were evaluated. At the end of 15 days of culture, no significant differences were observed among analyzed results of the different treatments. Rearing conditions were efficient, with high survival rates (average values superior than 91,6\%), and Artemia proved to be an attractive food. Salinity levels studied did not affect trairao larvae and fingerling development compared to freshwater.
\end{abstract}

Key Words: trairao, Hoplis lacerdae, larviculture, salinity, cannibalism, Artemia

\section{Introdução}

A larvicultura de peixes nativos com potencial para a piscicultura é, ainda hoje, fator de estrangulamento na criação de várias espécies. Essa dificuldade é relatada por vários autores (Basile-Martins, 1984; Castagnolli, 1992; Fregadolli, 1993; SipaúbaTavares \& Rocha, 1994), principalmente em relação às espécies que praticam o canibalismo durante toda a vida ou parte dela (Morais Filho \& Schubart, 1955; Pinto \& Guglielmoni, 1986; Cardoso et al., 1988; Zaniboni Filho \& Barbosa, 1992; Piovezan, 1994;
Cecarelli \& Volpato, 1996; Andrade-Talmelli, 1997; Luz, 2000; Luz et al., 2000a, Luz et al., 2000b). Neste sentido, existe a necessidade de busca de tecnologia específica para peixes nativos com potencial para a produção (Lopes et al., 1994).

A alimentação é um dos fatores de maior importância a serem considerados na fase de larvicultura (Basile-Martins, 1984; Sipaúba-Tavares \& Rocha 1994; Cestarolli et al., 1997), e a utilização de náuplios de Artemia é citada como uma ótima opção para algumas espécies brasileiras, como o pintado Pseudoplatystoma corruscans (Lopes et al., 1996;

\footnotetext{
${ }^{1}$ Eng.-Agrônomo, Doutorando Aqüicultura/UNESP, Bolsista/CAPES. E.mail: Iuzrk@ yahoo.com

2 Pesquisador Científico de Centro de Pesquisa em Reprodução e Larvicultura do Instituto de Pesca. Endereço: CAUNESP Via de acesso Prof. Paulo Donato Castellane, s/n. CEP 14884-900 Jaboticabal, SP, Brasil. E.mail: portella@caunesp.unesp.br
} 
Behr \& Hayashi, 1997; Leonardo et al., 2000), a piracanjuba Brycon orbignyanus (Piovezan, 1994), o cascudo Hoplosternum littorale (Ramnarine, 1994), o mandi-amarelo Pimelodus maculatus (Luz, 2000) e o pacu Piaractus mesopotamicus (Jomori, 2001). No entanto, este alimento constitui-se, num dos itens mais onerosos do sistema de produção de larvas (Jomori, 2001) e, por ser um organismo de água salgada, tem seu tempo de vida limitado em água doce. Esse fato acarreta a mortalidade dos náuplios, podendo provocar problemas de qualidade da água durante a larvicultura, além de limitar o tempo de exposição do alimento vivo às larvas.

Pesquisadores vêm realizando a larvicultura de espécies de água doce em meios ligeiramente salinizados (até 5\% de salinidade) para promover a sobrevivência dos náuplios de Artemia e melhorar as condições de cultivo (Lopes et al., 1996; Cestarolli \& Salles, 2000; Luz \& Zaniboni Filho, 2001). Wurts (1995) destaca a utilização de sal comum na água para reduzir a diferença osmótica com o meio externo e o estresse.

O trairão Hoplias lacerdae, espécie carnívora, nativa da bacia Amazônica, apresenta carne de excelente qualidade e características desejáveis para a pesca esportiva (Andrade et al., 1998). O canibalismo entre indivíduos dessa espécie é um dos maiores problemas enfrentados para a sua produção, estando relacionado principalmente à heterogeneidade de tamanho (Luz et al., 2000b). Em sistemas de produção em campo, com estocagem das larvas em tanques de cultivo adubados e com fornecimento de peixes forrageiros, observa-se sobrevivência variando de 25 a $75 \%$, ao final de 30 a 40 dias.

Desse modo, o objetivo desse trabalho foi avaliar e comparar a sobrevivência, o crescimento e a resistência ao estresse de larvas e alevinos de trairão Hoplias lacerdae, cultivados em água doce e salinizada, e alimentados com náuplios de Artemia como alimento vivo, durante os primeiros 15 dias de alimentação exógena.

\section{Material e Métodos}

Este experimento foi realizado no Laboratório de Nutrição de Organismos Aquáticos do CAUNESP, Jaboticabal, São Paulo.

Após a abertura da boca e inflação da vesícula gasosa, as larvas foram estocadas em 12 recipientes com volume útil de 1,5 L cada, dotados de sistema de aeração, numa densidade de 10 larvas/L. Para a manutenção da temperatura da água nos recipientes experimentais, eles foram distribuídos aleatoriamente dentro de três tanques de polietileno de $130 \mathrm{~L}$, com água suficiente para alcançar a metade da altura dos recipientes no sistema banho-maria. Em cada tanque, foi instalado um termostato para manter a temperatura da água em $29,5^{\circ} \mathrm{C}$.

No início do experimento, uma amostra de larvas foi sacrificada para a realização da biometria, quando foram avaliados o peso úmido e o comprimento total. Os tanques de polietileno foram totalmente cobertos com lona preta, mantendo-se o ambiente interno escuro, sendo descobertos somente para os manejos diários de alimentação e limpeza, por 10 minutos, aproximadamente.

As larvas foram submetidas a três tratamentos: cultivo em água doce; cultivo em água a $2 \%$ de salinidade; cultivo em água a $4 \%$ de salinidade. O delineamento experimental utilizado foi o inteiramente casualizado (DIC), constituído de três tratamentos e quatro repetições.

Para a salinização da água, foi utilizado sal grosso. O preparo dos meios foi realizado diariamente, sendo a salinidade aferida com o auxílio de um salinômetro.

A alimentação foi fornecida nas proporções diárias de 300 náuplios de Artemia/larva, do primeiro ao quinto dia, de 600 náuplios de Artemia/larva, do sexto ao décimo dia e de 900 náuplios de Artemial larva, do décimo primeiro ao décimo quinto dia. A quantidade diária de náuplios foi dividida em três refeições, fornecidas às 8,11 e $17 \mathrm{~h}$. A eclosão dos náuplios ocorreu diariamente em recipientes de $5 \mathrm{~L}$, utilizando-se de uma salinidade de $10 \%$.

Antes da última alimentação, era realizada a limpeza dos recipientes. Os detritos acumulados no fundo eram sifonados, e renovado cerca de $75 \%$ do volume de água, utilizando-se água com temperatura semelhante. Neste momento, era observada a ocorrência de mortalidade.

$\mathrm{O}$ pH da água foi medido a cada três dias, e a temperatura, diariamente. Ao final do experimento, após 15 dias, foram avaliados a taxa de sobrevivência e o crescimento. Esse último foi realizado através da medida do comprimento total (com paquímetro) e do peso úmido (em balança analítica) de cada alevino.

Com os resultados médios de peso inicial $\left(\mathrm{Wt}_{\mathrm{i}}\right) \mathrm{e}$ peso final $\left(\mathrm{Wt}_{\mathrm{f}}\right)$ de cada réplica, foi calculada a taxa de crescimento específico através da expressão $\mathrm{SGR}=100\left(\ln \mathrm{Wt}_{\mathrm{f}}-\ln \mathrm{Wt}_{\mathrm{i}}\right) / \Delta \mathrm{t}$, considerando $\Delta \mathrm{t} \mathrm{a}$ duração do experimento, em dias (Kestmont \& Stalmans, 1992). 


\section{Teste de resistência ao estresse}

De cada recipiente, foram coletados de seis a dez alevinos para a realização do teste de resistência ao estresse, segundo Atencio-Garcia (2000). Os alevinos foram capturados com peneira de malha de $0,5 \mathrm{~mm}$ e colocados em papel secante por 180 segundos, sendo depois recolocados nos mesmos recipientes e, durante duas horas, observados quanto ao comportamento e mortalidade.

Ao final desse período, foi verificado o estado físico dos indivíduos, considerando-se debilitados aqueles que se encontravam vivos, mas que apresentavam perda de postura no substrato dos aquários, e recuperados aqueles que se encontravam nadando na coluna d'água. Com esses dados, calculou-se a taxa de resistência ao estresse (\%).

\section{Análise estatística}

Os resultados de sobrevivência, peso, comprimento, taxa de crescimento específico e taxa de resistência ao estresse foram analisados, aplicando-se Análise de Variância paramétrica, pelo teste $\mathrm{F}$, considerando-se os resultados médios das larvas de cada parcela. Nos resultados em que foram verificadas diferenças significativas entre tratamentos $(\mathrm{P}<0,05)$, as médias foram comparadas pelo teste Tukey, a $5 \%$ de probabilidade. As taxas de sobrevivência, de crescimento específico e de resistência ao estresse sofreram transformação arco-seno.

\section{Resultados e Discussão}

O sistema de cultivo adotado (banho-maria) mostrou-se eficiente no controle da temperatura da água, não apresentando amplas variações $\left(29,5 \pm 0,5^{\circ} \mathrm{C}\right)$ entre os recipientes. $\mathrm{O}$ pH da água apresentou valores semelhantes em todos os tratamentos, com valor médio de 8,2 $\pm 0,2$. De acordo com Andrade et al. (1998), este peixe precisa de água com temperatura na faixa de 24,0 a $32,0^{\circ} \mathrm{C}$ e $\mathrm{pH}$, de 6 a 8 .

No início do experimento, quando estavam com oito dias de vida, as larvas apresentavam comprimento total médio de $9,8 \pm 0,2 \mathrm{~mm}$ e peso médio de 7,5 \pm $0,5 \mathrm{mg}$. Essa espécie apresenta desova parcelada, produzindo pequeno número de ovócitos por desova, porém com maior quantidade de vitelo, proporcionando um maior tempo de incubação e fase larval (Zaniboni Filho \& Barbosa, 1992). O maior tempo de período endotrófico pode ser considerado positivo ao seu cultivo, uma vez que permite melhor adequação das instalações, em função da disponibilidade de animais. O tamanho inicial das larvas é relativamente maior

R. Bras. Zootec., v.31, n.2, p.829-834, 2002 (suplemento) em comparação ao observado para outras espécies de peixes reofílicos descritas por Sato (1999).

Após 15 dias de tratamento, 23 após a eclosão, não foram verificadas diferenças significativas $(\mathrm{P}>0,05)$ para sobrevivência, peso, comprimento, taxa de crescimento específico e taxa de resistência ao estresse dos alevinos dos diferentes tratamentos (Tabela 1), indicando que os níveis de salinidade não afetaram o desenvolvimento das larvas e alevinos.

As altas taxas de sobrevivência observadas nos três tratamentos (Tabela 1) indicam que o sistema de cultivo (densidade de 10 larvas/L, ambiente interno escuro e alimentação com náuplios de Artemia, nas proporções utilizadas) mostrou-se eficiente para o desenvolvimento inicial desta espécie, considerada carnívora voraz e predadora visual (Lazzaro \& Ribeiro, 1984). Segundo Kubitza (1995), os principais problemas enfrentados na fase de larvicultura e alevinagem de espécies carnívoras são as inadequadas estratégias de manejo alimentar e de monitoramento e preparo dos tanques. Zaniboni Filho \& Barbosa (1992) relataram a larvicultura de trairão em condições controladas, utilizando zooplâncton coletado em tanque externo como alimento, por um período de 10 a 15 dias. Esses autores encontraram valores médios de sobrevivência e peso de $52 \%$ e 15,0 mg, respectivamente, salientando a ocorrência de canibalismo sob condições de elevadas densidades de estocagem ou de escassez de alimento. Resultados semelhantes de sobrevivência (média de 49,5\%) foram obtidos com a utilização de alimentação à base de zooplâncton selvagem durante as fases de larvicultura e alevinagem (Ribeiro \& Contijo, 1984). Entretanto, Sirol (1995), estudando larvas de trairão após a absorção total do vitelo (13,5 dias), estocadas na densidade de 15 larvas/L e alimentadas com náuplios de Artemia fornecidos ad libitum, por 10 dias, encontrou valores de sobrevivência superiores a 83,3\%.

Alevinos de trairão não apresentaram sinais ou sintomas de falta de adaptação às salinidades utilizadas, fato que permite a utilização de meios salinizados em sua larvicultura, no intuito de se evitar o aparecimento de fungos e doenças. Lopes et al. (1996) não observaram sintoma de falta de adaptação de larvas de pintado $P$. corruscans à salinidade de $4 \%$, durante os primeiros cinco dias de alimentação. No entanto, encontraram baixas taxas de sobrevivência, sendo a maior $(9,6 \%)$ observada entre as estocadas em menor densidade (30 larvas/litro) e alimentadas na combinação de náuplios de Artemia, rotífero marinho e cladócero de água doce. Cestarolli \& Sales (2000), 
Tabela 1 - Médias (土DP) de sobrevivência, peso, comprimento, taxa de crescimento específico e taxa de resistência ao estresse de alevinos de trairão (Hoplias lacerdae) após 15 dias de alimentação

Table 1 - Means $( \pm S D)$ for survival, weight, length, specific growth rate and stress resistence rate of trairao fingerlings (Hoplias lacerdae) at the end of 15 days of feeding

\begin{tabular}{lccc}
\hline $\begin{array}{l}\text { Tratamento } \\
\text { Treatment }\end{array}$ & $\begin{array}{c}\text { Água doce } \\
\text { Freshwater }\end{array}$ & $\begin{array}{c}\text { 2\%o de salinidade } \\
\text { 2\%osalinity }\end{array}$ & $\begin{array}{c}\text { 4\% de salinidade } \\
4 \% \text { salinity }\end{array}$ \\
\hline $\begin{array}{l}\text { Sobrevivência (\%) } \\
\text { Survival }\end{array}$ & $91,6 \pm 12,6$ & $100 \pm 0,0$ & $91,6 \pm 12,6$ \\
Peso (mg) & $122,0 \pm 25,0$ & $122,0 \pm 15,0$ & $131,0 \pm 21,0$ \\
Weight & & $22,9 \pm 1,1$ & $23,4 \pm 1,5$ \\
Comprimento(mm) & $22,4 \pm 1,7$ & $18,5 \pm 0,1$ & $19,1 \pm 0,7$ \\
Length & $18,6 \pm 0,8$ & 100,0 & 100,0 \\
SGR(\%) & 100,0 & & \\
$\operatorname{Re}(\%)$ & & &
\end{tabular}

SGR - Taxa de crescimento específico.

SGR - Specific growth rate.

Re - Taxa de resistência ao estresse.

$R e$ - Stress resistence rate.

avaliando a ingestão e taxa de alimentação em larvas de peixes tropicais tratados com náuplios de Artemia, utilizaram salinidade de $2 \%$ e não detectaram problemas aparentes para as larvas. Porém, Luz \& Zaniboni Filho (2001) observaram inchaço da região abdominal de larvas de mandi-amarelo, Pimelodus maculatus, cultivadas durante cinco dias em salinidade de $3 \%$ e alimentadas com náuplios de Artemia. Os autores verificaram melhor taxa de sobrevivência $(39,3 \%)$ e crescimento (6,3 mm de comprimento total e $0,9 \mathrm{mg}$ de peso) para larvas cultivadas em água doce. Já os alevinos de bagre, ojundiá(Rhamdia quelen), toleraram salinidades de $9 \%$ com água doce salinizada com sal marinho, e de 10\%o com água do mar, durante 96 horas, conforme observado por Marchioro e Baldisseroto (1999).

Em relação à larvicultura em água doce de outras espécies de peixes carnívoros, utilizando náuplios de Artemia como alimento vivo, destacamse as pesquisas de Behr \& Hayashi (1997) com larvas de $P$. corruscans, estocadas na densidade de 10 larvas/litro, com resultados de sobrevivência, peso e comprimento de $65,6 \%, 15,0 \mathrm{mg}$ e 14,0 mm, respectivamente, após nove dias de cultivo. A larvicultura de dourado, Salminus maxillosus, foi estudada por Luz et al. (2000a), que avaliaram larvas mantidas na densidade de 30 larvas/litro, e alimentadas diariamente com 40 náuplios de Artemial larva, durante quatro dias. Os autores verificaram sobrevivência média de $4,8 \%$ e valores de peso e comprimento de 5,7 mg e 9,5 mm, respectivamente. Comparando-se os resultados obtidos no presente trabalho (Tabela 1), com os de outras espécies carnívoras, infere-se que a larvicultura do trairão foi bem-sucedida.

Segundo Hecht \& Pienaar (1993), o canibalismo durante a larvicultura pode ser controlado através de várias técnicas, como a alimentação até a saciedade, freqüência ótima de alimentação, tamanho apropriado do alimento, distribuição homogênea do alimento, uso preferencial de alimento vivo e densidades de estocagem adequadas. Diariamente, no momento da retirada da lona para a alimentação, as larvas que se encontravam na coluna d'água, desciam para o fundo dos recipientes, permanecendo aglomeradas próximo à pedra porosa, em busca de abrigo. Contudo, logo após o fornecimento do alimento vivo, movimentavam-se ativamente em busca dos náuplios, indicando que esse alimento é atrativo. Observou-se, também, que os peixes apresentavam trato digestório alaranjado pela presença dos náuplios.

A utilização do sistema de escuridão total durante a larvicultura parece positiva para esta espécie, quando associada aos demais manejos utilizados. Britz \& Pienaar (1992) relataram para o cultivo de alevinos de Clarias gariepinus, em regime de 24 horas de escuro, redução do estresse, da agressão e do canibalismo, além de aumento no crescimento. Segundo Pienaar (1990), o canibalismo pode estar relacionado à turbidez da água, intensidade de luz e existência de refúgios.

No momento da limpeza dos recipientes, não foram observados larvas ou alevinos mortos. Assim, o desaparecimento de larvas e alevinos foi atribuído ao canibalismo, sendo, porém, observado $100 \%$ de sobrevivência em várias unidades experimentais. Não 
foram verificadas sobras de alimento, mostrando que não foi fornecido em quantidades excessivas, fator importante para a manutenção da qualidade da água.

De acordo com Verreth \& Bieman (1987), a taxa de crescimento específico pode ser utilizada para comparar o crescimento de larvas, sendo afetada pela temperatura e pelo nível de alimentação. Os autores encontraram valores de crescimento variando de 40,7 a 41,9\%, em função da temperatura da água no cultivo de larvas de Clarias gariepinus. Esta taxa de crescimento também pode variar em função da densidade de estocagem e do sistema de cultivo adotado (Hossain et al., 1998). Atencio-García (2000) também encontrou alta taxa de crescimento específico $(36,8 \%)$ em larvas de yamú Brycon siebenthalae, que atingiram $29,1 \mathrm{~mm}$ de comprimento total quando cultivadas durante 15 dias em viveiros de terra. Os valores da taxa de crescimento específico encontrados para o trairão, durante os 15 primeiros dias de alimentação exógena, foram inferiores aos citados acima, porém os meios utilizados não influenciaram $(\mathrm{P}>0,05)$ esses valores (Tabela 1$)$.

Após o teste de estresse, observou-se que os alevinos apresentavam perda de postura (natação errática) quando recolocados nos recipientes, reestabelecendo-a após cinco a 10 minutos. Os alevinos que se encontravam no fundo dos recipientes apoiados lateralmente, e não nadavam ativamente na coluna da água, foram considerados debilitados $(10,1 \pm 14,4 \% ; 17,5 \pm 17,1 \% ; 25,2 \pm 14,2 \%$, para os tratamentos em água doce, 2 e $4 \%$ o de salinidade, respectivamente). Porém, quando os recipientes foram agitados, apresentavam movimentos natatórios, não sendo verificada mortalidade após duas horas de observação, em todas as réplicas (Tabela 1).

O teste de resistência ao estresse, mantendo-se os animais fora da água por um determinado tempo, tem sido utilizado para avaliar a qualidade nutricional dos alimentos ingeridos (Kraul et al., 1993; Watanabe $\&$ Kiron, 1994). No manejo de primeira alimentação de larvas de yamú $B$. siebenthalae, alimentadas com diferentes organismos, o fornecimento de náuplios de Artemia ou larvas de pirapitinga Piaractus brachypomus proporcionou indivíduos mais resistentes ao estresse, comparativamente aos que receberam zooplâncton silvestre e aos mantidas em jejum (Atencio-García, 2000). No presente estudo, em que os alevinos de trairão receberam o mesmo manejo alimentar, com o objetivo de avaliar o efeito do meio de cultivo na resistência ao estresse, encontrou-se $100 \%$ de sobrevivência em todos os tratamentos.

\section{Conclusões}

A larvicultura de trairão Hoplias lacerdae, durante os primeiros 15 dias de alimentação exógena, pode ser realizada tanto em água doce, como em água salinizada a 2 e $4 \%$, sem prejudicar o desenvolvimento das larvas e alevinos.

A utilização de náuplios de Artemia mostrou-se um alimento atrativo e eficiente para o desenvolvimento inicial das larvas e alevinos de trairão.

Os manejos de cultivo adotados durante a fase de larvicultura foram adequados para a espécie, possibilitando altas taxas de sobrevivência.

\section{Literatura Citada}

ANDRADE, D.R.; VIDAL, M.V.J.; SHIMODA, E. Criação do trairão Hoplias lacerdae. Universidade Estadual do Norte Fluminense-UENF, 1998. 32p. (Boletim Técnico, v.3)

ANDRADE-TALMELLI, E.F. Indução reprodutiva e ontogenia inicial da piabanha Brycon insignis (Steindachner, 1876) (Characiformes, Bryconinae), mantida em confinamento - Vale do Paraíba, SP. São Carlos: Universidade Federal de São Carlos, 1997. 185p. Dissertação (Mestrado em Ecologia e Recursos Naturais) - Universidade Federal de São Carlos, 1997.

ATENCIO-GARCÍA, V.J. Influência da primeira alimentação na alevinagem do yamú Brycon siebenthalae (EIGENMANN, 1912). Florianópolis: Universidade Federal de Santa Catarina, 2000. 130p. Dissertação (Mestrado em Aqüicultura) - Universidade Federal de Santa Catarina, 2000.

BASILE-MARTINS, M.A. Criação de organismos para alimentação de larvas de peixes. In: SIMPÓSIO BRASILEIRO DE AQÜICULTURA, 1984, São Carlos. Anais... São Carlos, 1984. p.97-100.

BEHR, E.R.; HAYASHI, C. Alimentação de larvas de Pseudoplatystoma corruscans (Agassiz, 1829) em bandejas berçário durante o período crítico. In: ENCONTRO BRASILEIRO DE ICTIOLOGIA, 12., 1997, São Paulo. Resumos... São Paulo: Sociedade Brasileira de Ictiologia, 1997. p.51.

BRITZ, P.J.; PIENAAR, A.G. Laboratory experiments on the effect of light and cover on the behaviour and growth of African catfish Clarias gariepinus (Pisces:Clariidae). Journal of Zoology, v.227, p.43-62, 1992.

CARDOSO, E.L.; FERREIRA, R.M.A.; ALVES, M.S.D. Desenvolvimento embrionário e estádios larvários inicias em surubim (Pseudoplatystoma corruscans Agassz, 1829). In: VI ENCONTRO ANUAL DE AQÜICULTURA - ASSOCIAÇÃO MINEIRA DE AQÜICULTURA, Belo Horizonte, 1988. Resumos... Belo Horizonte: 1988. p.22.

CASTAGNOLLI, N. Piscicultura de água doce. Jaboticabal: FUNEP. 1992. 189p.

CECCARELLI, P.S.; VOLPATO, G.L. Canibalismo em larvas de matrinxã, Brycon cephalus: Efeito da densidade e de consorciação com pacu e curimbatá. In: SIMPÓSIO BRASILEIRO DE AQÜICULTURA, 10., 1996, Sete Lagoas. Resumos... Sete Lagoas: 1996. p.78.

CESTAROLLI, M.A.; PORTELLA, M.C.; ROJAS, N.E.T. Efeito do nível de alimentação e do tipo de alimento na sobrevivência e no desempenho inicial de larvas de Curimbatá Prochilodus scrofa (STEINDACHNER, 1881). Boletim do Instituto de Pesca, v.24 (único), p.119-129, 1997. 
CESTAROLLI, M.A.; SALLES, F.A. Ingestão e taxa de alimentação em larvas de peixes tropicais de água criados em laboratório. In: SIMPÓSIO BRASILEIRO DE AQÜICUlTURA, 11., 2000, Florianópolis. Anais... Florianópolis, 2000. CD-ROM. Nutrição. 14.

FREGADOLLI, C.H. Seleção alimentar de larvas de pacu Piaractus mesopotamicus Holmberg, 1887 e tambaqui Colossoma macropomum Cuvier, 1818, em laboratório. Boletim Técnico CEPTA , v.6, n.1, p.1-50, 1993.

HECHT, T.; PIENAAR, A. A review of cannibalism and its implications in fish larviculture. Journal of World Aquaculture Society, v.24, n.2, p.247-261, 1993.

HOSSAIN, M.A.R.; BEVERIDGE, M.C.M.; HAYLOR, G.S. The effects of density, light and shelter on the growth and survival of African catfish (Clarias gariepinus Burchell, 1822) fingerlings. Aquaculture, v.160, p.251-258, 1998.

JOMORI, R.K. Desenvolvimento, sobrevivência e aspectos econômicos da produção de alevinos de pacu, Piaractus mesopotamicus (Holmberg, 1887), diretamente em viveiros ou com diferentes períodos de larvicultura em laboratório. Jaboticabal: Universidade Estadual Paulista, 2001. 69p. Dissertação (Mestrado em Aqüicultura) - Universidade Estadual Paulista, 2001.

KESTMONT, P.; STALMANS, J.M. Initial feeding of European minnow larvae Phoxinus phoxinus L. 1. Influence of diet and feeding level. Aquaculture, v.104, p.327-340, 1992.

KRAUL, S.; AKO, H.; CANTRELL, R. et al. Nutritional factors affecting stress resistence in the larval mahimahi, Coryphaena hippurus. Journal of World Aquaculture Society, v.24 n.2, p.186-193, 1993.

KUBITZA, F. Preparo de rações e estratégias de alimentação no cultivo intensivo de peixes carnívoros. In: SIMPÓSIO INTERNACIONAL SOBRE NUTRIÇÃO DE PEIXES E CRUSTÁCEOS, Campos de Jordão, 1995. Anais... Campos de Jordão: 1995. p.91-115.

LAZZARO, X.; RIBEIRO, D.M. Comportamento alimentar, seletividade e taxa de alimentação das larvas de Hoplias lacerdae (Erytrinidae; Trairão). In: ENCONTRO ANUAL DE AQÜICULTURA DE MINAS GERAIS, 3., Igarapé, 1984. Resumos... Igarapé: 1984. p.13-14.

LEONARDO, A.F.G.; ASSAL, J.F.; MOKI, D.A. et al. Efeito da alimentação natural e artificial na larvicultura de pintado Pseudoplatystoma corruscans. In: SIMPÓSIO BRASILEIRO DE AQÜICULTURA, 11., Florianópoils: 2000. Resumos... Florianópolis, 2000. CD-ROM. Nutrição. 25.

LOPES, R.N.M.; FREIRE, R.A.B.; VICENSOTTO, J.R.M. et al. Alimentação de larvas de surubim Peseudoplatystoma corruscans (AGASSIZ, 1829) em laboratório na primeira semana de vida. Boletim Técnico CEPTA, v.9, p.11-29, 1996.

LOPES, R.N.M.; SENHORINI, J.A.; SOARES, M.C.F. Crescimento e sobrevivência de larvas de matrinxã Brycon cephalus Gunther, 1869, (Pisces, Characidae) sob diferentes dietas alimentares. Boletim Técnico CEPTA, v.7, p.41-48, 1994.

LUZ, R.K. Larvicultura do mandi-amarelo Pimelodus maculatus (Lacépède 1803): desenvolvimento embrionário, larval e primeira alimentação. Florianópolis: Universidade Federal de Santa Catarina, 2000. 47p. Dissertação (Mestrado em Aqüicultura) - Universidade Federal de Santa Catarina, 2000.

LUZ, R.K.; FERREIRA, A.A.; REYNALTE-TATAJE, D.A. et al. Larvicultura de dourado (Salminus maxillosus, Valenciennes, 1849), nos primeiros dias de vida. In: SIMPÓSIO BRASILEIRO DE AQÜICULTURA, 1., 2000, Florianópolis. Anais...Florianópolis: 2000a. CD-ROM. Peixes. 28.

LUZ, R.K.; SALARO, A.L.; SOUTO, E.F. et al. Avaliação de canibalismo e comportamento territorial de alevinos de trairão (Hoplias lacerdae). Acta Scientiarum, v.22, n.2, p.465-469, 2000b.

R. Bras. Zootec., v.31, n.2, p.829-834, 2002 (suplemento)
LUZ, R.K.; ZANIBONI FILHO, E. Influência da salinidade nos primeiros dias de vida do mandi-amarelo Pimelodus maculatus. In: ENCONTRO BRASILEIRO DE ICTIOLOGIA, 14., 2001, São Leopoldo. Resumos.... São Leopoldo: 2001. CDROM. Fisiologia. 08luzron01.

MARCHIORO, M.I.; BALDISSEROTO, B. Sobrevivência de alevinos de jundiá (Rhamdia quelen Quoy \& Gaimard, 1824) à variação de salinidade da água. Ciência Rural, v.29, n.2, p.315-318, 1999.

MORAIS FILHO, M.B.; SCHUBART, O. Contribuição ao Estudo do dourado, Salminus maxillosus Val., do Rio Mogi Guaçu (Pisces, Characidae). São Paulo: Ministério da Agricultura, 1955. 131p.

PIENAAR, A.G. A study of coeval sibling cannibalism in larval and juvenile fishes and its control under culture conditions. Grahamstown, South Africa: Rhodes University, 1990. 162p. Thesis (Master) - Rhodes University, 1990.

PINTO, M.L.; GUGLIELMONI, L.A. Observações sobre o desenvolvimento e comportamento alimentar das larvas de dourado, Salminus maxillosus (VALENCIENNES, 1894). In: SIMPÓSIO BRASILEIRO DE AQÜICULTURA, 4., 1986, Cuiabá. Anais... Cuiabá: 1986. p.35-47.

PIOVEZAN, U. Efeito da dieta na sobrevivência de larvas de piracanjuba (Brycon orbignyanus) - CAUNESP. In: SEMINÁRIO SOBRE CRIAÇÃ̃O DE ESPÉCIES DO GÊNERO Brycon, 1., 1994, Pirassununga. Anais... Pirassununga: 1994. p. $17-18$.

RAMNARINE, I.W. Larval culture, development and growth of the cascudo, Hoplosternum littorale (Hancock 1828, Callichthyidae). Aquaculture, v.126, p.291-298.

RIBEIRO, D.M.; GONTIJO, V.P.M. Reprodução de trairão em cativeiro. Informe Agropecuário, v.10, n.110, p.21-25, 1984.

SATO, Y. Reprodução de peixes da Bacia do Rio São Francisco: Indução e caracterização de padrões. São Carlos: Universidade Federal de São Carlos, 1999. 179p. Tese (Doutorado em Ecologia e Recursos Naturais) - Universidade Federal de São Carlos, 1999.

SIPAÚBA-TAVARES, L.H.; ROCHA, O. Sobrevivência de larvas de Piaractus mesopotamicus (Holmberg, 1887) (Pacu) e Colossoma macropomum (Cuvier, 1818) (Tambaqui), cultivadas em laboratório. Biotemas, v.7, n.1/2, p.46-56, 1994.

SIROL, R.N. Efeito da inanição sobre o desenvolvimento inicial da larva de Hoplias cf. lacerdae Ribeiro, 1908 (Characiformes: Erytrinidae). Viçosa, MG: Universidade Federal de Viçosa, 1995. 61p. Dissertação (Mestrado em Zootecnia) - Universidade Federal de Viçosa, 1995.

VERRETH, J.; BIEMAN, H.D. Quantitative feed requirements of African catfish (Clarias gariepinus Burchell) larvae fed with descapsulated cystis of Artemia. Aquaculture, v.63, p.251-267, 1987.

WATANABE, T.; KIRON, V. Prospects in larval fish dietetics. Aquaculture, v.24, p.223-251, 1994.

WURTS, W.A. Using salt to reduce handling stress in channel catfish. World Aquaculture, v.26, n.3, p.80-81, 1995.

ZANIBONI FILHO, E.; BARBOSA, N.D.C. Larvicultura na CEMIG. In: ENCONTRO ANUAL DE AQÜICULTURA DE MINAS GERAIS, 10., 1992, Belo Horizonte. Anais...Belo Horizonte: 1992. v.10. p.36-42.

Recebido em: 12/09/01

Aceito em: 05/04/02 